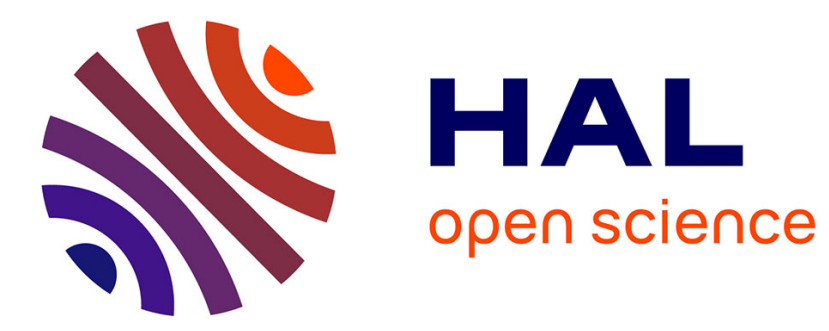

\title{
Non-linear piezoelectric effect in CdTe and CdZnTe
}

\author{
R. André, C. Bodin, Joel Cibert, L. Dang, G. Feuillet
}

\section{To cite this version:}

R. André, C. Bodin, Joel Cibert, L. Dang, G. Feuillet. Non-linear piezoelectric effect in CdTe and CdZnTe. Journal de Physique IV Proceedings, 1993, 03 (C5), pp.C5-429-C5-432. 10.1051/jp4:1993592 . jpa-00251679

\section{HAL Id: jpa-00251679 https://hal.science/jpa-00251679}

Submitted on 1 Jan 1993

HAL is a multi-disciplinary open access archive for the deposit and dissemination of scientific research documents, whether they are published or not. The documents may come from teaching and research institutions in France or abroad, or from public or private research centers.
L'archive ouverte pluridisciplinaire HAL, est destinée au dépôt et à la diffusion de documents scientifiques de niveau recherche, publiés ou non, émanant des établissements d'enseignement et de recherche français ou étrangers, des laboratoires publics ou privés. 


\title{
Non-linear piezoelectric effect in CdTe and CdZnTe
}

\author{
R. ANDRÉ, C. BODIN, J. CIBERT, L.S. DANG and G. FEUILLET*
}

Laboratoire de Spectrométrie Physique, URA 08 du CNRS, Université Joseph Fourier, Grenoble I, BP. 87, 38402 Saint Martin d'Hères cedex, France

* DRFMC - SP2M - PSC, Centre d'Etudes Nucléaires de Grenoble, BP. 85X, 38041 Grenoble cedex, France

\begin{abstract}
Strained quantum wells (QWs) grown along the polar axis (111) in a zincblende semiconductor exhibit a piezoelectric field. This field strongly modifies the energy of excitons confined in QWs. We have extracted, from optical measurements of excitonic transitions, the piezoelectric field value in a large set of QWs: $\mathrm{CdTe} / \mathrm{CdZnTe}$, CdTe/CdMnTe, CdZnTe/CdMnTe. By suitably choosing the composition of alloys, we have obtained different values of strains (compressive or extensive) due to the lattice mismatch between QWs and barriers. The non-linear piezoelectric effect appears clearly when plotting the piezoelectric tensor component $\mathrm{e}_{14}$ versus lattice mismatch. For example $\mathrm{e}_{14}$ increases from $0.07 \mathrm{C} / \mathrm{m}^{2}$ to $0.15 \mathrm{C} / \mathrm{m}^{2}$ when the lattice mismatch varies from $-0.3 \%$ to $-1.7 \%$.
\end{abstract}

\section{INTRODUCTION.}

Strained heterostructures of semiconductors grown along a polar crystallographic axis exhibit a piezoelectric field which deeply modifies their band configuration and their optical properties. Such structures are of particular interest for device applications in non-linear optics, for example to design optical switches. Piezoelectric field in strained heterostructures has been theoretically predicted by Smith and Mailhiot $[1,2]$ and then experimentally proved $[3,4,5]$. Fields in excess of $10^{5} \mathrm{~V} / \mathrm{cm}$ are experimentally observed in $\Pi I-\mathrm{V}$ and II-VI $[6,7]$ heterostructures strained by about $1 \%$. A rather surprising result is the observation of a nonlinear piezoelectric effect in compressively strained CdTe QWs [8]. In this paper we have extended the study of the piezoelectric effect to QWs under both compressive and extensive strains. The piezoelectric coefficient $\mathrm{e}_{14}$ is found to be different from the value in the literature $[9,10]$ and a strong variation of $\mathrm{e}_{14}$ with the strain state is again observed which cannot be fully explained by recent theoretical calculations.

\section{EXPERIMENTS.}

\subsection{Samples.}

Lattice parameters of $\mathrm{CdTe}, \mathrm{MnTe}$ and $\mathrm{ZnTe}$ are $6.48 \AA, 6.33 \AA$ and $6.10 \AA$ respectively [11]. For alloys $\left(\mathrm{Cd}_{1-x} \mathrm{Mn}_{\mathrm{x}} \mathrm{Te}\right.$ and $\left.\mathrm{Cd}_{1-\mathrm{x}} \mathrm{Zn} \mathrm{n}_{\mathrm{x}} \mathrm{Te}\right)$ we assume, according to Végard law, a linear dependence of the lattice parameter " $a$ " on the composition " $x$ ". Samples are elaborated by molecular beam epitaxy on a vicinal (111) $\mathrm{Cd}_{0.96} \mathrm{Zn}_{0.04} \mathrm{Te}$ substrate. QWs are coherently grown on a buffer layer $(-3 \mu \mathrm{m})$, thick enough to be nearly strain-free. Barriers have the same composition as the buffer layer: thus barriers are strain-free and exhibit no piezoelectric field whereas wells are under biaxial strain due to the lattice mismatch. Three types of samples have been grown: $\mathrm{CdTe} / \mathrm{CdZnTe}$ and $\mathrm{CdTe} / \mathrm{CdMnTe}$ for which $\mathrm{CdTe}$ is always under compressive strain, and $\mathrm{CdZnTe} / \mathrm{CdMnTe}(\sim 10 \% \mathrm{Zn})$ for which the in plane strain $\varepsilon_{/ / \Gamma}\left(a_{\text {well }}-a_{\text {parries }}\right) / a_{\text {well }}$ can be made positive (extensive strains) or nearly equal to zero. By suitably choosing the 
composition of alloys, we have obtained fifteen samples for which QWs are under different strain states ranging from $\boldsymbol{E}_{/ /}=-1.7 \%$ to $\boldsymbol{E}_{/ /}+0.2 \%$. Each sample contains at least three QWs of different thicknesses, which allows a direct determination of the internal electric field (section 3.1). Moreover a cap layer of about $3000 \AA$ was used to remove the QWs from the region of surface field (see below).

\subsection{Optical experiments}

Optical properties of strained QWs were investigated by reflectivity, photoluminescence (PL) and photoluminescence excitation at pumped helium temperature. To avoid any screening of piezoelectric field, the photoluminescence excitation power was kept below $1 \mathrm{~W} / \mathrm{cm}^{2}$. The alloy composition of the barrier CdZnTe or CdMnTe was determined from optical measurement of its excitonic gap. Applying the Vegard law one deduces its lattice parameter, and thus the in-plane strain $\varepsilon_{/ /}$in the QWs.

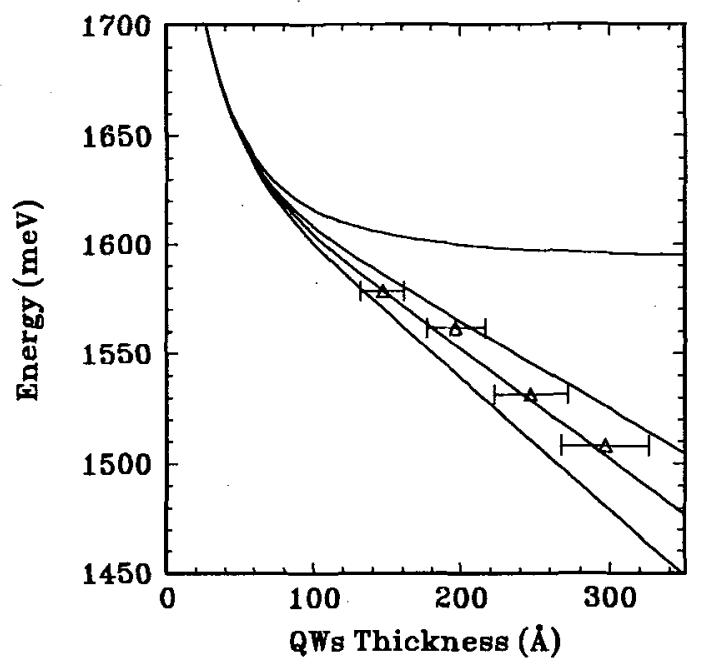

\subsection{Surface field}

A series of samples were grown to estimate the surface electric field effect which could alter the internal piezoelectric field. These

Figure 1: Photoluminescence energy of four piezoelectric QWs $\mathrm{CdTe} / \mathrm{Cd}_{0.87} \mathrm{Mn}_{0.13} \mathrm{Te}$ (triangles) of different thicknesses (all other parameters being equal) and calculated curves corresponding, in order of decreasing energy, to $F=0,0.4,0.5$ and $0.6 \mathrm{mV} / \AA$.

samples contained two identical QWs. The first QW was grown deep below the surface $(\sim 1$ $\mu \mathrm{m})$ and served as a reference. The second QW was grown at different depths (from 170 to $1300 \AA)$ to probe the surface effect. The electric field continuously decreases inside the sample. The experimental results are consistent with a simple model [12] assuming a depletion region of width $2500 \AA$ and a constant impurities density of $4.10^{15} \mathrm{~cm}^{-3}$. Within this model the maximum electric field was found to be about $0.2 \mathrm{mV} / \AA$ at the surface and it vanishes at a depth of $2500 \AA$. A piezoelectric $\mathrm{QW}$ is a very good probe for this study because its fundamental energy level is sensitive to first order to little variations of the electric field whereas the same variations will affect a square $Q W$ only to second order.

\section{RESULTS AND DISCUSSION}

\subsection{Determination of the electric field}

The energy levels in piezoelectric QWs have been calculated within the envelope function approximation, assuming a flat band configuration for the barriers. Figure 1 shows a fit for a sample containing four QWs of different thicknesses. Since these QWs were grown on the same buffer layer they experience the same in plane-strain $E_{/ /}$and therefore the same piezoelectric field. This reduces the number of free parameters for the fit to one (the field $F$ ). Figure 2 shows a plot of the measured fields in QWs under compressive and extensive strain. For comparison, we have plotted the piezoelectric field expected from linear elasticity and piezoelectricity:

$$
F=\frac{2 \sqrt{3}}{\varepsilon \varepsilon_{0}} \frac{c_{11}+2 c_{12}}{c_{11}+2 c_{12}+4 c_{44}} e_{14} \varepsilon_{/ /}
$$


$\mathrm{c}_{\mathrm{ij}}$ are elasticity tensor components and $\varepsilon \varepsilon_{0}$ is the permittivity of the material. Taken into account $c_{i j}$ values from references $[14,15,16]$ and the bulk value of $e_{14}$ determined by piezoelectrically active shear waves $[9,10]$ $\left(\mathrm{e}_{14}=0.0335 \mathrm{C} / \mathrm{m}^{2}\right), \mathbf{F}$ should follow the dash line on figure 2 . Clearly other effects than linear piezoelectricity are involved, such as interface charges or a non linear piezoelectric effect. Charges at (111) interfaces are not ruled out by symmetry, but they are calculated to be very small in GaAs/AlAs [17]. Moreover the measured field does not depend on the nature of the barriers (CdMnTe or $\mathrm{CdZnTe}$ ). Hence, in the following, we make the hypothesis of a non-linear piezoelectric effect.

\subsection{Non-linearities.}

There are three possible sources for non-linearity in equation (1): $\varepsilon, c_{i j}$ and $e_{14}$. The fields involved in this study are rather small (a few $\mathrm{mV} / \AA$ ), so the linear regime should be appropriate for the dielectric constant $\varepsilon[18]$. Non-linearity of elastic constants have been investigated under high hydrostatic pressure by Maheswaranathan et al. [16]. According to their results, in the range of strains in our QWs, non-linearity of $c_{i j}$ is about $2 \%$ and may be neglected. Abinitio calculation of $\mathrm{c}_{\mathrm{ij}}$ [18] leads to the same conclusion. So, non-linearity of the electric field could only be attribufed to the piezoelectricity tensor component $e_{14}$. Equation (1) has been used to extract $e_{14}$ from the measured electric field and the result is shown in figure 3 . Since the sign of $\mathrm{F}$ cannot be measured in our experiments, we have arbitrarily chosen the sign of $\mathrm{e}_{14}$ to get a smooth variation when going from negative to positive strain. Not only $e_{14}$ value is different from the value obtained in bulk materials by ultrasonic waves determination $[9,10]$, but it also largely depends on strain.

Piezoelectricity in zinc-blende materials has been theoretically investigated by Martin [19] and Hübner [20]. In their models, the piezoelectric constant involves an ionic contribution, related to the internal displacement, and an electronic contribution. In CdTe, these contributions are almost equal in magnitude but opposite in sign. This situation is particularly favourable to non linear effects. A recent work by $\mathrm{Dal}$ Corso et al. [18] provides an interesting ab-initio calculation of $\mathrm{e}_{14}$ for CdTe using the density functional theory. They report that non linear piezoelectricity in $\mathrm{CdTe}$ is mainly a volume effect and obtain a linear variation of $\mathrm{e}_{14}$ with the change of volume. In (111) strained QWs the change of volume can be expressed in terms of the in-plane strain $\varepsilon_{/ /}$:

$$
\frac{\delta V}{V}=\frac{12 C_{44}}{C_{11}+2 C_{12}+4 C_{44}} \varepsilon_{/ /}
$$

Using this relation we have compared our results to the calculation of Dal Corso et al. The predicted variation of $\mathrm{e}_{14}$ is too weak to account for the strong increase we observed for 
$\left|\varepsilon_{/ /}\right|<0.5 \%$. On the other hand, the experimental $\mathrm{e}_{14}$ seems to follow the predicted linear dependence for $\varepsilon_{/ /}<-0.5 \%$.

\section{CONCLUSION}

We have observed a strong dependence of $e_{14}$ with strains due to lattice mismatch in (111) oriented QWs. This is due to the fact that $e_{14}$ results from two contributions (ionic and electronic) of opposite sign which nearly cancel in CdTe. The non linearity of piezoelectricity is not fully explained by the volume effect mentioned in recent ab-initio calculations. Further investigations under high hydrostatic pressure are planned.

\section{REFERENCES}

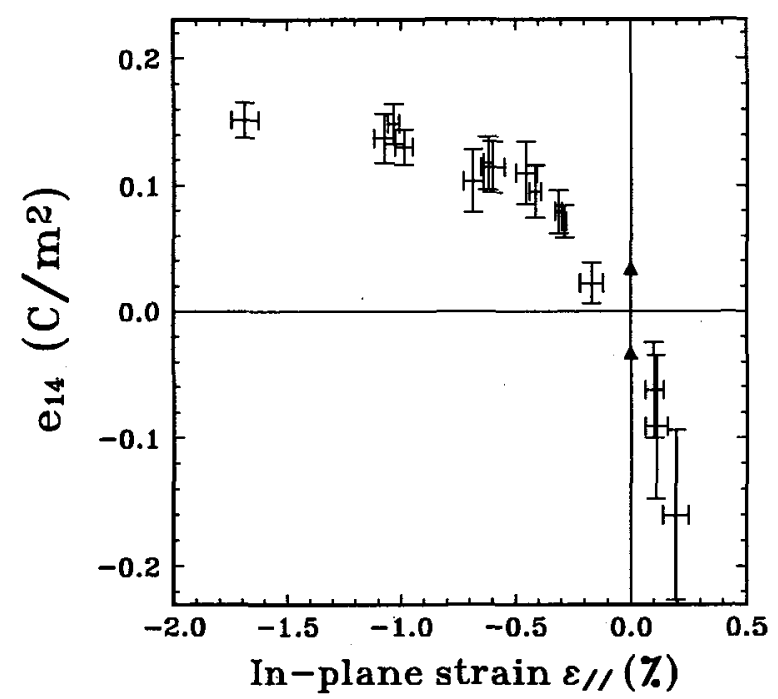

Figure 3: Piezoelectricity tensor component $e_{14}$ extracted from piezoelectric $Q W s$ under different strain states. Triangles are the absolute value of $\mathrm{e}_{14}$ taken in the literature $[9,10]$.

[1] D.L. Smith, Solid State Commun. 57 (1986) 919.

[2] D.L. Smith and C. Mailhiot, Rev. Mod. Phys. 62 (1990) 173.

[3] B.K. Laurich, K. Elcess, C.G. Fonstad, J.G.Beery, C. Mailhot and D.L. Smith, Phys. Rev. Lett. 62 (1989) 649.

[4] I. Sela, D.E. Watkins, B.K. Laurich, D.L. Smith, S. Subbanna and H. Kroemer, Appl. Phys. Lett. 58 (1991) 684.

[5] K.W. Goossen, E.A. Caridi, T.Y. Chang, J.B. Stark, D.A.B. Miller, and R.A. Morgan, Appl. Phys. Lett. 56 (1990) 715.

[6] R. André, C. Deshayes, J. Cibert, Le Si Dang, S. Tatarenko and K. Saminadayar, Phys. Rev. B 42 (1990) 11392.

[7] Le Si Dang, R. André, C. Bodin-Deshayes, J. Cibert, H. Okumura, G. Feuillet and P.H. Jouneau, Physica B 185 (1993) 551.

[8] J. Cibert, R. André, C. Deshayes, Le Si Dang, H. Okumura, S. Tatarenko, G. Feuillet, P.H. Jouneau, R. Mallard and K.Saminadayar, J. of Crystal Growth 117 (1992) 424.

[9] D. Berlincourt, H. Jaffe and L.R. Shiozawa, Phys. Rev. 119 (1963) 1009.

[10]P.Maheswaranathan, R.J. Sladek and U. Debska, Phys. Rev.B 31 (1985) 7910.

[11]Landolt-Bornstein, Numerical Data and Functional Relationships in Science and Technology, Vol.22 Semiconductors (Springer Verlag Berlin Heidelberg 1987).

J.K. Furdyna, J. Appl. Phys. 64 (1988) R29.

[12]R.A. Smith, Semiconductors, (Cambridge University Press, 2nd Ed, 1978) pp 219-222.

[13] J.F. Nye, Physical properties of crystals, (Oxford University Press, 1967) pp 110-149.

[14]H.J. McSkimin and D.G. Thomas, J. of appl. phys. 33 (1962) 56.

[15] R.D. Greenough and S.B. Palmer, J. Phys. D: APPL. Phys. 6 (1973) 587.

[16]P.Maheswaranathan, R.J. Sladek and U. Debska, Phys. Rev.B 31 (1985) 5212.

[17]N. Chetty and R.M. Martin, Phys Rev B 45 (1992) 6089, and references therein.

[18] Andrea Dal Corso, Raffaele Resta and Stephano Baroni, Phys. Rev B (1993) in press.

[19]R.M. Martin, Phys. Rev. B 5 (1972) 1607.

[20]K. Hübner, Phys. Stat. Sol. (b) 57 (1973) 627. 